\title{
Characterization of the Asian Citrus Psyllid Transcriptome
}

\author{
Justin Reese ${ }^{1, * 凶}$, Matthew K. Christenson, ${ }^{2,}$, Nan Leng 3 , Surya Saha4, Brandi Cantarel ${ }^{1}$, Magdalen \\ Lindeberg ${ }^{4}$, Cecilia Tamborindeguy ${ }^{5}$, Justin MacCarthy ${ }^{1}$, Daniel Weaver ${ }^{1}$, Andrew J. Trease ${ }^{2}$, Steven V. \\ Ready $^{2}$, Vincent M. Davis ${ }^{6}$, Courtney McCormick ${ }^{3}$, Christian Haudenschild ${ }^{3}$, Shunsheng Han ${ }^{7}$, Shannon L. \\ Johnson7, Kent S. Shelby ${ }^{8}$, Hong Huang ${ }^{9}$, Blake R. Bextine ${ }^{10}$, Robert G. Shatters'11, David G. Hall11, Paul H. \\ Davis2,12 and Wayne B. Hunter11 \\ 1. Genformatic, LLC, 6301 Highland Hills Drive Austin, TX 78731, USA. \\ 2. Department of Biology, University of Nebraska at Omaha, Omaha, Nebraska 68182, USA. \\ 3. Illumina, Inc., 25861 Industrial Blvd, Hayward, California 94545, USA. \\ 4. Department of Plant Pathology and Plant-Microbe Biology, Cornell University, Ithaca New York 14853, USA. \\ 5. Department of Entomology, Texas A\&M University, 2475 TAMU College Station, Texas 77843, USA. \\ 6. Heteroskedastic, Inc., Arvada, Colorado, USA. \\ 7. Los Alamos National Laboratory Bioscience division B-6, MS M888 Los Alamos, New Mexico 87545, USA. \\ 8. USDA Agricultural Research Service, 1503 South Providence Road, Columbia, Missouri 65203, USA. \\ 9. School of Information, University South Florida, 4202 East Fowler Avenue, Tampa, FL 33260, USA. \\ 10. University of Texas at Tyler, 3900 University Boulevard, Tyler, TX, 75799, USA. \\ 11. USDA Agricultural Research Service, 2001 South Rock Road, Fort Pierce, FL 34945, USA. \\ 12. Department of Genetics, Cell Biology and Anatomy, University of Nebraska Medical Center, Omaha, Nebraska 68182, USA.
}

* These authors contributed equally to this work.

$\bowtie$ Corresponding author: jreese@genformatic.com (JR); wayne.hunter@ars.usda.gov (WBH).

( ) Ivyspring International Publisher. This is an open-access article distributed under the terms of the Creative Commons License (http://creativecommons.org/ licenses/by-nc-nd/3.0/). Reproduction is permitted for personal, noncommercial use, provided that the article is in whole, unmodified, and properly cited.

Published: 2014.02.10

\begin{abstract}
The Asian citrus psyllid, Diaphorina citri Kuwayama (Hemiptera: Psyllidae) is a vector for the causative agents of Huanglongbing, which threatens citrus production worldwide. This study reports and discusses the first $D$. citri transcriptomes, encompassing the three main life stages of $D$. citri, egg, nymph and adult. The transcriptomes were annotated using Gene Ontology (GO) and insecticide-related genes within each life stage were identified to aid the development of future $D$. citri insecticides. Transcriptome assemblies and other sequence data are available for download at the International Asian Citrus Psyllid Genome Consortium website [http://psyllid.org/download] and at NCBI [http://www.ncbi.nlm.nih.gov/bioproject/29447].
\end{abstract}

Key words: Asian Citrus Psyllid, Diaphorina citri Kuwayama

\section{Results and Discussion}

\section{General Characteristics of the D. citri Tran- scriptomes}

In total, 46,927,970 reads of $75 \mathrm{bp}, 39,830,860$ reads of $110 \mathrm{bp}$, and 50,248,212 reads of $100 \mathrm{bp}$ were generated from the egg, nymph, and adult tissues, respectively, comprising $12.9 \mathrm{~Gb}$ of sequence, and were used to construct three de novo stage-specific transcriptomes (Table 1). The GC content of the transcriptomes was highly similar, ranging from $42.10 \%$ to $44.81 \%$. Moreover, 99 to $100 \%$ of core eukaryotic genes had a detectable homolog in the stage-specific assemblies and between 84.3 and $88.2 \%$ of core genes had a detectable homolog whose alignment covered 
$>80 \%$ of the core gene, indicating the transcriptomes were fairly complete.

\section{Functional Annotation and Characterization of Diaphorina citri Transcripts}

To review the putative functions of the $D$. citri transcripts and validate the completeness of the transcriptomes, GO analysis was performed against the three $D$. citri stages, plus four related organisms with complete transcriptome information. Of the total number of representative transcripts present within the egg, nymph and adult stages, $45.20 \%(26,546)$, $46.29 \%(26,651)$, and $40.00 \%(21,218)$ were assigned GO terms, respectively (Figure 1). Importantly, all four related organisms displayed this pattern, suggesting the completeness of the three stage transcriptomes.

\section{Identification of Diaphorina citri Insecti- cide-Related Genes}

Insecticides are a pivotal component in controlling $D$. citri populations throughout the world, and therefore, it is essential to develop potent insecticides against a wide variety of molecular $D$. citri targets [1]. Insecticide targets and genes involved in detoxification and resistance were identified in the egg, nymph, and adult stages (Figure 2A). The largest and most complex group was 'Juvenile Hormone Metabolism.' Putative $D$. citri homologs of the major gene products involved in juvenile hormone metabolism exist within each life stage and exhibit a high degree of similarity to those genes of closely related organisms (Figure 2B and Table 2).

Table I. Statistics for Diaphorina citri transcriptome assemblies.

\begin{tabular}{|c|c|c|c|c|c|c|c|c|}
\hline Assembly & Stage & $\begin{array}{l}\text { Total } \\
\text { Number of } \\
\text { Transcripts }\end{array}$ & $\begin{array}{l}\text { Mean } \\
\text { Contig } \\
\text { Length }\end{array}$ & GC $\%$ & $\begin{array}{l}\text { Core Eukaryotic } \\
\text { Genes with } \\
\text { Homologs }\end{array}$ & $\begin{array}{l}\text { Core Eukaryotic Genes } \\
\text { with Homologs ( }>80 \% \\
\text { coverage) }\end{array}$ & $\begin{array}{l}\% \text { of Transcripts } \\
\text { Confirmed in } \\
\text { Draft Genome } \\
\text { Assembly }\end{array}$ & $\begin{array}{l}\text { Putative Gene } \\
\text { Fusions }\end{array}$ \\
\hline Complete & Egg & 76,550 & 852 & 42.1 & $458 / 458(100 \%)$ & $400 / 458(87.3 \%)$ & $81.6 \%$ & $196(0.256 \%)$ \\
\hline- & Nymph & 69,233 & 756 & 44.81 & $456 / 458(99.56 \%)$ & $404 / 458(88.2 \%)$ & $81.0 \%$ & $146(0.211 \%)$ \\
\hline- & Adult & 62,450 & 691 & 42.96 & $458 / 458(100 \%)$ & $386 / 458(84.3 \%)$ & $81.0 \%$ & $158(0.253 \%)$ \\
\hline Representative* & Egg & 58,814 & 719 & 41.83 & $458 / 458(100 \%)$ & $400 / 458(87.3 \%)$ & $84.2 \%$ & $163(0.278 \%)$ \\
\hline- & Nymph & 57,635 & 632 & 44.27 & $456 / 458$ (99.56\%) & $403 / 458(88.0 \%)$ & $79.0 \%$ & $123(0.214 \%)$ \\
\hline - & Adult & 53,117 & 595 & 42.67 & $458 / 458(100 \%)$ & $386 / 458(84.3 \%)$ & $88.7 \%$ & 147 (0.277\%) \\
\hline
\end{tabular}

Files containing the assembled transcripts for each of the D. citri life stages were analyzed using custom Perl scripts, and the total number of transcripts, mean contig length, $\%$ GC, number of core eukaryotic genes, \% transcripts detectable in the genome assembly and number of putative gene fusions were determined. (See Methods section for details about how each was calculated.)

Table 2. Juvenile hormone related genes identified in the Diaphorina citri transcriptome.

\begin{tabular}{|c|c|c|c|c|c|c|c|}
\hline Gene Name & $\begin{array}{l}\text { NCBI } \\
\text { Gene ID }\end{array}$ & FlyBase ID & Query ID & $\begin{array}{l}\text { Query } \\
\text { Length (bp) }\end{array}$ & Subject ID & Subject Species & E-Value \\
\hline Allatostatin & 42947 & FBgn0015591 & diaci_nymph_66660000039047 & 692 & NP_001037036.1 & Bombyx mori & $3 e-07$ \\
\hline Allatostatin Receptor & 44126 & FBgn0028961 & diaci_nymph_66660000026007 & 1547 & NP_524700.1 & Drosophila melanogaster & 0 \\
\hline Broad & 44505 & FBgn0000210 & diaci_nymph_66660000033288 & 578 & |NP_726750.1 & Drosophila melanogaster & 0 \\
\hline Chd64 & 38490 & FBgn0035499 & diaci_nymph_66660000018386 & 2227 & AAF47840.2 & Drosophila melanogaster & $1 e-136$ \\
\hline $\begin{array}{l}\text { Cytosolic Juvenile Hormone } \\
\text { Binding Protein }\end{array}$ & 733092 & - & diaci_nymph_66660000009996 & 768 & NP_001037668.1 & Bombyx mori & $4 e-142$ \\
\hline FKBP39 & 41860 & FBgn0013269 & diaci_nymph_66660000005954 & 2150 & CAA86996.1 & Drosophila melanogaster & 0 \\
\hline Hexamerin & 660274 & - & diaci_nymph_66660000015491 & 2322 & NP_001164358.1 & Tribolium castaneum & 0 \\
\hline $\begin{array}{l}\text { Juvenile Hormone Acid } \\
\text { Methytransferase }\end{array}$ & 34977 & FBgn0028841 & diaci_egg_55550000038216 & 1502 & EFA02917.1 & Tribolium castaneum & $3 e-126$ \\
\hline $\begin{array}{l}\text { Juvenile Hormone Epoxide } \\
\text { Hydrolase }\end{array}$ & 251984 & FBgn0010053 & diaci_nymph_66660000003152 & 1779 & NP_001161902.1 & Tribolium castaneum & $2 \mathrm{e}-49$ \\
\hline Juvenile Hormone Esterase & 36780 & FBgn0010052 & diaci_adult_77770000038704 & 271 & NP_001180223.1 & Tribolium castaneum & $9 e-84$ \\
\hline $\begin{array}{l}\text { Juvenile Hormone Esterase } \\
\text { Binding Protein }\end{array}$ & 37996 & FBgn0035088 & diaci_nymph_66660000032724 & 733 & NP_611989.1 & Drosophila melanogaster & $2 \mathrm{e}-18$ \\
\hline Methoprene-Tolerant & 32114 & FBgn0002723 & diaci_egg_55550000026684 & 389 & ABR25244.1 & Tribolium castaneum & $8 e-39$ \\
\hline Retinoid X Receptor & 31165 & FBgn0003964 & diaci_nymph_66660000021934 & 2841 & AAF45707.1 & Drosophila melanogaster & $5 e-93$ \\
\hline
\end{tabular}

Putative juvenile hormone related genes were identified using BLASTX to compare the representative $D$. citri transcripts from the egg, nymph and adult life stages to a custom BLAST database containing those genes of interest from closely related organisms. The top BLAST hits for the juvenile hormone related genes are shown. 


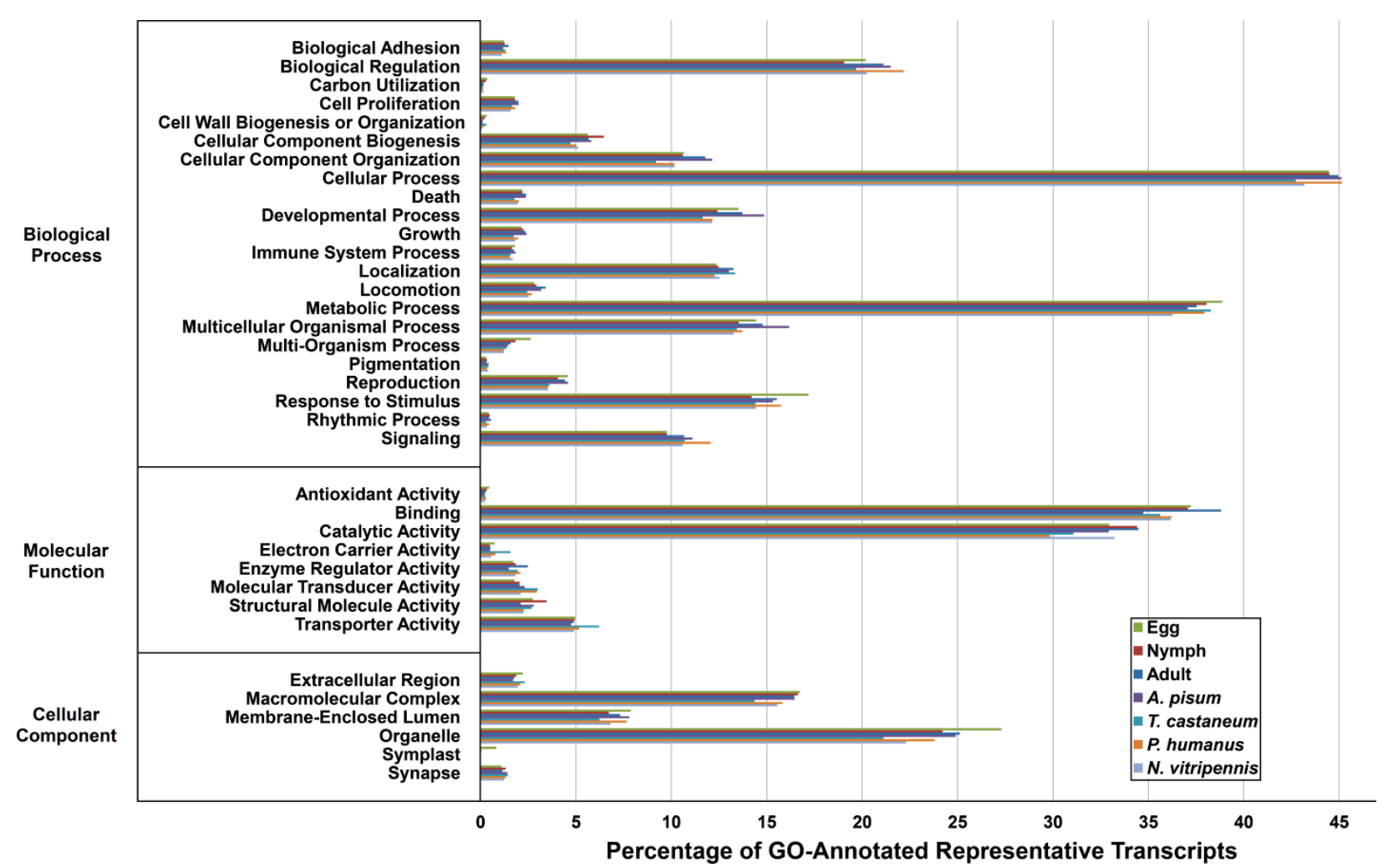

Figure I. Gene Ontology (GO) analysis of the representative transcripts present within the three life stages of Diaphorina citri. GO-terms were assigned to the representative transcripts of the three D. citri life stages and Acyrthosiphon pisum, Tribolium castaneum, Pediculus humanus and Nasonia vitripennis, the four organisms that exhibited the greatest number of top BLAST hits, using B2G4Pipe and then sorted into groups within three independent compartments using Blast2GO. The 'Cellular Process', 'Binding' and 'Organelle' groups contain the greatest number of representative transcripts within the 'Biological Processes', 'Molecular Function' and 'Cellular Component' domains, respectively. The representative transcriptomes of the four organisms and the egg, nymph and adult stages exhibited similar patterns, and thus indicates the completeness of the stage transcriptomes.

A

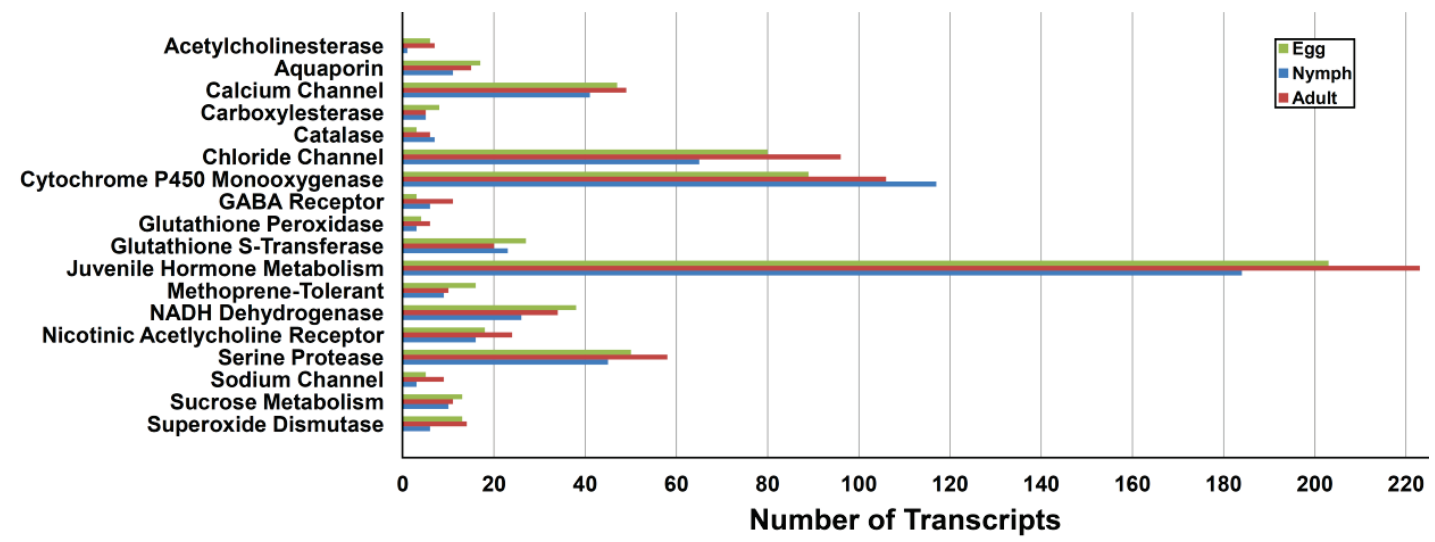

B

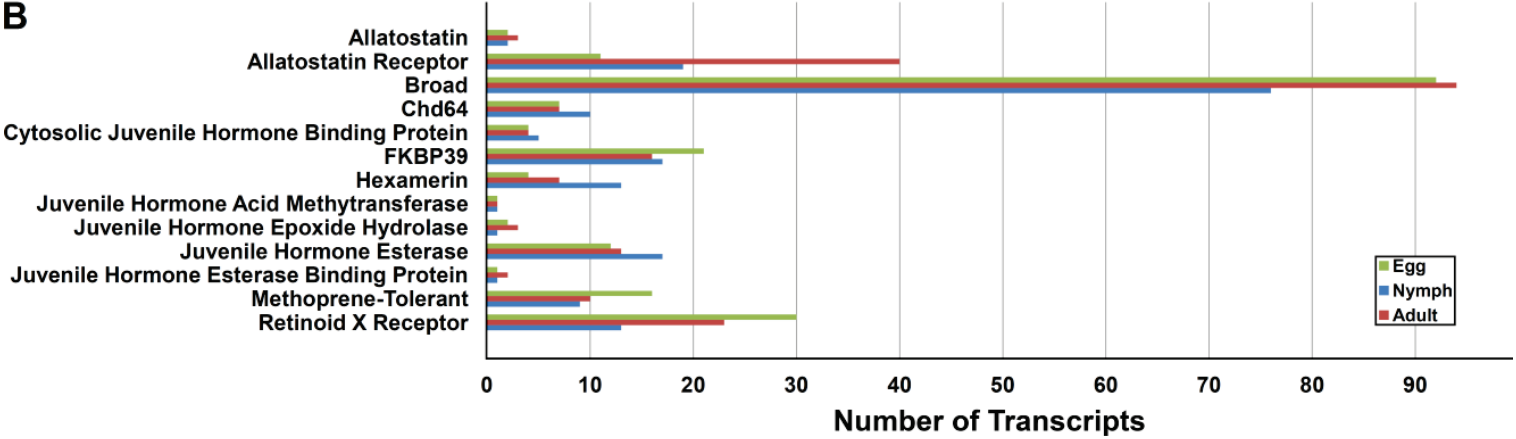

Figure 2. Genes related to insecticide targets, detoxification, and resistance within each of the three life stages of Diaphorina citri. Identification of putative $D$. citri insecticide-related genes was accomplished by comparing the representative transcripts from the three $D$. citri life stages to a custom protein database containing homologous genes of interest from closely related organisms using BLASTX. (A) D. citri predicted gene products of putative insecticide targets and predicted gene products putatively involved in detoxification and resistance were identified in all three life stages. (B) $D$. citri homologs of the major gene products involved in insect juvenile hormone metabolism are present within each life stage. 


\section{Methods}

\section{Growth, Sample Preparation, and Sequence Generation}

Psyllids were field collected from citrus groves near USDA-ARS research station, 2001 South Rock Road, Fort Pierce, FL, 34945-3030; no specific permits were required. Psyllids were reared in $2^{\prime} \times 2^{\prime}$ cages, temperature maintained at $26^{\circ} \mathrm{C}$, lighting was dependent on natural day length throughout the year, and fed on Murraya paniculata for two years then Citrus macrophylla for the last two years. Tissue from one to four day old eggs, 3rd and 4th instar nymphs, and one day to one month old adults of mixed genders were collected, processed, and held at $-80^{\circ} \mathrm{C}$ until RNA isolation.

Total RNA was isolated from whole egg, nymph, and adult tissues using Qiagen's RNeasy Mini Kit, per the manufacturer's instructions. The mRNA was purified using poly-T oligo-attached magnetic beads and converted into CDNA with random primers using the mRNA sequencing preparation kit from Illumina (part number 1004898). The cDNA was sequenced using an Illumina GAIIx sequencing system.

\section{Transcriptome Assembly and Characteriza- tion}

Velvet (v1.0.19) (k-mer of 47) and Oases (v0.1.19) were used to generate three stage-specific transcriptomes using reads from the egg, nymph or adult tissues, respectively [2,3]. Contigs with adapter sequence contamination were removed by alignment to Illumina adapter sequences using BWA [4].

The GC content and mean transcript length, were calculated using a custom Perl script to check completeness, transcriptomes were aligned with TBLASTN to sequences representing 458 core eukaryotic genes [5], and percent of core genes with at least one KOG family member that aligned to a transcript with an E-value < 1e-6 (optionally with alignment that covered $80 \%$ of KOG family member) was measured. To detect gene fusions, transcripts were aligned using BLASTN to 16,172 gene predictions produced using MAKER [134], and considered gene fusions if 1) the alignments between the transcript and two different MAKER gene predictions had an E-value $<1$ e- 6 and percent identity $>95 \%, 2$ ) coordinates of the two alignments on the transcript overlapped by $<10 \mathrm{nu}-$ cleotides, 3) the two alignments together covered $>95 \%$ of transcript sequence and 4) no alignment existed between transcript and MAKER model with an $E$-value $<1$ e- 6 and percent identity $>95 \%$ that covered $>95 \%$ of the transcript.

\section{Generation of Representative Transcriptome Transcripts}

To generate transcriptomes containing only unique transcripts for downstream analysis, by removing all repetitive, identical and near-identical transcripts, CD-HIT-EST was used with a sequence identity cut-off of $99 \%$ [6,7]. A loss of $23.17 \%(17,736)$, $16.75 \%(11,598)$, and $14.94 \%(9,333)$ of the total transcripts from the egg, nymph, and adult stages, respectively, was observed. Also, in order to compare $D$. citri to other previously sequenced Insecta spp., all NCBI RefSeq nucleotide records (retrieved August 12, 2012) were collected for Acyrthosiphon pisum, Tribolium castaneum, Pediculus humanus and Nasonia vitripennis totaling 17,$675 ; 10,417 ; 10,775$ and 12,927 sequences, respectively. In order to generate representative transcriptomes for each of these species, near-identical transcripts were removed using CD-HIT-EST using a cut-off value of $99 \%[6,7]$. This resulted in a $4.64 \%$ (821), $4.09 \%$ (426), $0.47 \%$ (51) and $2.07 \%$ (267) loss of the total number of transcripts for A. pisum, T. castaneum, P. humanus and $N$. vitripennis, respectively.

\section{Gene Ontology}

To infer the putative function of the $D$. citri transcripts, BLASTX and an E-value threshold of $\leq$ 1e-5 was used to scan the representative transcriptomes against the NCBI non-redundant (nr) database (retrieved April 23, 2012). GO terms were assigned and were then categorized using the programs B2G4Pipe (version 2.5.0) and Blast2GO (version 2.5.1) using default settings, respectively, and the b2g_may12 GO database [8].

\section{Identification of Insecticide-Related Genes}

Insecticide-related genes present within the different life stages of $D$. citri were identified by using a combination of BLASTN, TBLAST, and BLASTX and an $E$-value threshold of $\leq 1 \mathrm{e}-5$ to compare the representative transcriptomes to a custom BLAST database containing insecticide-related genes from A. pisum, Apis mellifera, Bombyx mori, Drosophila melanogaster, $N$. vitripennis, P. humanus, and T. castaneum (retrieved from NCBI on July 30, 2012).

\section{Acknowledgements}

This publication was supported by grants from the NIH National Center for Research Resources (5P20RR016469) and the National Institute for General Medical Science (8P20GM103427), USDA-ARS U.S. Horticultural Research Lab, Subtropical Insects Research Unit, Ft. Pierce, FL and a grant from the Citrus Research Board, Inc. (217 North Encina, P.O. Box 230, Visalia, CA. 93279). Its contents are the sole responsi- 
bility of the authors and do not necessarily represent the official views of NIH, NIGMS, USDA or CRB. Additional support was provided by FIRE and FUSE grants from the University of Nebraska at Omaha. This work utilized the Holland Computing Center of the University of Nebraska. We also acknowledge Biological Technicians Maria T. Gonzalez, Belkis Diego, Kathy Moulton, Ashley Voss, Carol Malone, PeiLing Li, USDA-ARS, U.S. Horticultural Research Laboratory, Fort Pierce, FL, 34945; Technicians: Chloe Hawkings, Evelien Van Ekert, Sulley K. Ben-Mahmoud, Erica R. Egan, Lindsay Shaffer, University of Florida-IFAS, IRREC, Fort Pierce, FL 34945; Tim Crouch, Associate Director, Networks and Operations, University of Texas at Tyler, TX; and Goutam Gupta, Los Alamos National Laboratory, Los Alamos, NM 87545. We acknowledge Angela Douglas and Xiangfeng Jing, Department of Entomology, Cornell University, NY, 14853 for the osmoregulatory analysis, and Dr. Xiomara Sinisterra, Science, Biotechnology Advisor, University of Texas at Tyler, TX, and Kevin Hodges.

\section{Competing Interests}

The authors have declared that no competing interests exist.

\section{References}

1. Hall, DG, Ammar ED, Richardson ML, and Halbert SE. Asian citrus psyllid, Diaphorina citri (Hemiptera: Psyllidae), vector of citrus huanglongbing disease. Entomologia Experimentalis et Applicata 2012; 146: 207-223.

2. Schulz MH, Zerbino DR, Vingron M, Birney E. Oases: robust de novo RNA-seq assembly across the dynamic range of expression levels. Bioinformatics 2012; 28: 1086-1092.

3. Zerbino DR, Birney E. Velvet: algorithms for de novo short read assembly using de Bruijn graphs. Genome Res 2008; 18: 821-829.

4. $\mathrm{Li} \mathrm{H}$, Durbin R. Fast and accurate short read alignment with Burrows-Wheeler transform. Bioinformatics 2009; 25: 1754-1760.

5. Parra G, Bradnam K, Korf I. CEGMA: a pipeline to accurately annotate core genes in eukaryotic genomes. Bioinformatics 2007; 23: 1061-1067.

6. Li W, Godzik A. Cd-hit: a fast program for clustering and comparing large sets of protein or nucleotide sequences. Bioinformatics 2006; 22: 1658-1659.

7. Limin Fu, Beifang Niu, Zhengwei Zhu, Sitao $\mathrm{Wu}$ and Weizhong Li. CD-HIT: accelerated for clustering the next generation sequencing data. $\begin{array}{lllll}\text { Bioinformatics 2012; } 28 & \text { (23): } & 3150-3152 . & \end{array}$ 10.1093 /bioinformatics/bts565.

8. Conesa A, Gotz S, Garcia-Gomez JM, Terol J, Talon M, et al. Blast2GO: a universal tool for annotation, visualization and analysis in functional genomics research. Bioinformatics 2005; 21: 3674-3676. 